\title{
Intrahepatic insulin resistance in a murine model of steatohepatitis: effect of PPAR $\gamma$ agonist pioglitazone
}

\author{
Isabelle A Leclercq, Valérie A Lebrun, Peter Stärkel and Yves J Horsmans \\ Laboratory of Gastroenterology, Faculty of Medicine, Université catholique de Louvain (UCL), Brussels, \\ Belgium
}

\begin{abstract}
Hepatic insulin resistance is associated with hepatic steatosis and is thought to play an important role in the pathogenesis of steatohepatitis. Using a murine model of steatohepatitis (mice fed a diet deficient in methionine and choline-MCD diet), we tested the effects of the insulin-sensitising, PPAR $\gamma$ agonist drug pioglitazone (PGZ) on systemic and intrahepatic insulin sensitivity and on liver pathology. Compared to controls, mice fed the MCD diet develop a significant steatohepatitis, have enhanced glucose tolerance and enhanced systemic response to insulin. PGZ did not affect the systemic insulin sensitivity in control or MCD-fed mice as assessed in vivo by intraperitoneal glucose or insulin dynamic tests. However, PGZ prevented hepatic fat accumulation and steatohepatitis induced by the MCD diet. This effect was associated with an increased mass of adipose tissue and increased expression and release of adiponectin, while hepatic acyl co-enzyme A oxidase and acyl-coenzyme A carboxylase, regulating hepatic $\beta$-oxidation of fatty acid, remained unchanged. Steatohepatitis in MCD-diet-fed mice was associated with intrahepatic insulin resistance as shown by a reduced phosphorylation of hepatic insulin receptor, and Akt in response to an insulin stimulus. PGZ to MCD-fed mice restored the activation of the insulin receptor and of the Akt pathway in response to insulin. In conclusion, PGZ alleviates steatosis and steatohepatitis induced by the MCD diet, an effect associated with correction of intrahepatic insulin resistance.
\end{abstract}

Laboratory Investigation (2007) 87, 56-65. doi:10.1038/labinvest.3700489; published online 30 October 2006

Keywords: nonalcoholic steatohepatitis; hepatic insulin resistance; pioglitazone; PPARgamma

The thiazolidinediones (TZDs) are a class of antidiabetic drugs which allow the normalisation of blood glucose without a concomitant risk of hypoglycaemia. $^{1,2}$ Their potent insulin-sensitising effect is mediated via ligand activation of transcription factor peroxisome proliferator-activated receptor gamma (PPAR $\gamma)$, which controls transcription of genes involved in lipid metabolism, glucose transport and metabolism, energy balance and production of adipokines such as adiponectin and TNF. ${ }^{2-5}$ Despite the wide usage of these drugs, the respective roles of various PPAR $\gamma$-expressing target cell types and the precise molecular mechanisms underlying TZD action are not completely understood..$^{2,6}$ PPAR $\gamma$ is abundantly expressed in adipocytes and is

Correspondence: Dr IA Leclercq, MD, PhD, Laboratoire de Gastroentérologie, Faculty of Medicine, Université catholique de Louvain (UCL), GAEN 53/79, Avenue Mounier, 53, B-1200 Brussels, Belgium.

E-mail: isabelle.leclercq@gaen.ucl.ac.be

Received 19 May 2006; revised and accepted 15 September 2006; published online 30 October 2006 important for adipocyte differentiation. ${ }^{7}$ Adipocytes are believed to be the primary site of action of TZDs. ${ }^{8,9}$ However, in animal models of lipoatrophy, TZDs still have beneficial insulin-sensitising effects in the near absence of adipose tissue, ${ }^{10}$ suggesting that PPAR $\gamma$ agonists may act directly on non-adipose tissues. ${ }^{11}$ Targeted deletion of PPAR $\gamma$ in muscle leads to insulin resistance. ${ }^{12,13}$ Mice without liver PPAR $\gamma$ also develop insulin resistance, but respond to TZDs by lowering serum glucose and insulin levels and improved glucose clearance. ${ }^{10}$ It is, therefore, unclear whether PPAR $\gamma$ in liver and muscle are direct target for TZDs action or whether the antidiabetic effects in liver and muscle are secondary to TZD action in adipocytes through induction of an adipocyte-derived hormonal factor. Beside, PPAR $\gamma$ is also expressed in other cell types such as macrophages, vascular endothelial cells or hepatic stellate cells suggesting a more pleiotropic role in multiple fundamental pathways as diverse as the control of inflammatory reaction (which can also in turn influence insulin sensitivity), atherosclerosis or hepatic fibrosis. ${ }^{14-17}$ 
Nonalcoholic fatty liver disease (NAFLD) is one of the most common cause of liver disease worldwide. ${ }^{18}$ The two basic histological lesions in subjects with NAFLD are steatosis and steatohepatitis (termed non-alcoholic steatohepatitis or NASH), the latter being characterised by the presence of hepatocellular damage, inflammation and progressive fibrosis. ${ }^{19}$ The pathogenesis of NASH remains ill-defined. The prominent association with insulin resistance suggests that this condition may contribute to the pathogenesis of NAFLD by promoting the development of steatosis and the subsequent cell injury and inflammation leading to $\mathrm{NASH}^{20}$

Insulin resistance increases peripheral lipolysis and delivery of free fatty acids to the liver. It is associated with hyperinsulinemia, which stimulates intrahepatic lipogenesis, and thereby induces intrahepatic lipid storage (steatosis). The liver is a target organ for insulin. Binding of insulin to its receptor (IR) induces its autophosphorylation, which, via phosphorylation of insulin receptor substrate (IRS) proteins, activates two main signalling pathways. The PI3K/Akt pathway is responsible for most of the metabolic actions of insulin governing hepatic storage and release of glucose according to the metabolic needs, and the ras-mitogen-activated protein kinase (MAPK) pathways controls cell growth and proliferation. Resistance in the metabolic pathway results in persistence of hepatic glucose output in the face of hyperinsulinemia, which participates in the maintenance of hyperglycemia and resulting stimulation of insulin secretion, and to the aggravation of insulin resistance. ${ }^{21}$ Hepatic insulin resistance is associated with the development of hepatic steatosis, but the link between the two remains unclear. ${ }^{22}$ Some have postulated that the combination of insulin resistance, hyperglycemia and increased concentrations in circulating fatty acids promote hepatic lipid synthesis and impair hepatic $\beta$-oxidation leading to hepatic steatosis. ${ }^{22,23}$ Others have proposed that hepatic fat accumulation and hepatic insulin resistance can occur without the development of peripheral (or systemic) insulin resistance. ${ }^{24-26}$ In addition, while impaired regulation of hepatic glucose output is documented in patients with $\mathrm{NASH},{ }^{23}$ it is still ill-defined whether and how impaired hepatic insulin signalling is implicated in the promotion and the progression of steatohepatitis.

Mice fed a lipogenic methionine and choline deficient (MCD) diet develop a steatohepatitis mimicking hepatic lesions seen in patients with NASH. ${ }^{27-32}$ However, contrasting with obesity and metabolic syndrome associated with human NASH, MCD-fed mice lose weight and do not have systemic (or peripheral) insulin resistance. ${ }^{33}$ Despite this, a recent study suggests that they exhibit intrahepatic resistance to the action of insulin. ${ }^{34}$ PPAR $\gamma$ agonists TZDs have been shown to improve hepatic steatosis in several hepatic disease models in rodent. The aim of this study was to analyse the effect of the treatment by the TZD pioglitazone (PGZ) on liver pathology in MCD-fed mice, and to analyse the consequences of this on systemic and intrahepatic response to insulin.

\section{Materials and methods}

\section{Animals and Experimental Protocols}

Female C57BL6/J mice (8-10 weeks old) were randomly divided into four experimental groups and fed a powdered (a) lipogenic MCD diet (cat. no. 960439, ICN, Aurora, OH, USA), (b) MCD diet supplemented with PGZ at $0.01 \%(\mathrm{w} / \mathrm{w})$ (gift from Takeda, Japan), (c) control diet (MCD diet supplemented with DL-methionine $(3 \mathrm{~g} / \mathrm{kg})$ and choline chloride (2 g/kg); cat. no. 960441 , ICN) or (d) control diet supplemented with PGZ at $0.01 \%(\mathrm{w} / \mathrm{w})$. Mice (five per group) were allowed food and water ad libitum for 4 weeks. All studies were approved by the local Ethics Committee for Animal Use. At the end of the feeding experiment, mice were euthanised, blood collected and livers excised. A part of the right posterior lobe was fixed in $10 \%$ formalin. The remaining liver was snap frozen. Subcutaneous fat from the abdominal area was removed and weighted.

In a separate experiment and after completion of a 4-week feeding protocol, mice from the 4 dietary groups were either injected insulin ( $5 \mathrm{U} ; n=4$ per treatment group) or a similar volume of PBS $(n=4$ per treatment group) in the portal vein, 5 min before the resection of the liver, to analyse the intrahepatic response to insulin.

\section{Morphological Studies}

Sections ( $4 \mu \mathrm{m}$ thick) from formalin-fixed liver tissue were stained with haematoxylin and eosin. Hepatic inflammation was quantified as the ratio of the area of inflammatory foci (defined as groups of five or more inflammatory cells) to the total area of the section. ${ }^{28}$ Two different sections of each liver were examined on coded slides.

\section{Biochemical Assays}

Serum alanine aminotransferase (ALT) levels were measured using automated techniques. Hepatic total lipid content was measured as described. ${ }^{27}$ Insulin, leptin and adiponectin serum concentrations were determined using commercial mouse ELISA or radio-immunoassays (Linco Research, St Charles, MI, USA). At 1 week before harvest, mice were tested for their tolerance to glucose and their response to insulin. Glucose $(1.5 \mathrm{~g} / \mathrm{kg}$ body weight) was injected i.p. on $4 \mathrm{~h}$ fasting animals and glucose levels were monitored on tail blood 0, 15, 30, 60, 90 and $120 \mathrm{~min}$ after the injection. Systemic response 
to insulin was assessed by monitoring blood glucose levels $0,15,30,45,60$ and $120 \mathrm{~min}$ after the injection of insulin ( $0.5 \mathrm{U} / \mathrm{kg}$ body weight i.p.) administered after overnight fasting.

\section{Immunoquantitation of Proteins}

Liver homogenates were prepared in a buffer containing Triton X-100 0.1\%, Hepes $50 \mathrm{mM}, \mathrm{NaCl}$ $150 \mathrm{mM}$, Glycerol $10 \%, \mathrm{MgCl}_{2} 1.5 \mathrm{mM}$, DTT $0.5 \mathrm{mM}$, PMSF $125 \mu \mathrm{M}$, aprotinin $50 \mu \mathrm{g} / \mathrm{ml}$, leupeptin $5 \mu \mathrm{g} / \mathrm{ml}$, $\mathrm{NaF} 0.5 \mathrm{mM}, \mathrm{NaVO}_{4} 50 \mu \mathrm{M}$ and used in Western blot assays. Total and phosphorylated forms of IR, IRS1, IRS2, Akt, extracellular signal-regulated kinase (ERK) 1/2 (p42/44), Acyl co-enzyme A-carboxylase (ACC) were assayed by Western immunoblots. After separation by SDS-PAGE, proteins were transferred onto PVDF membrane (NEN Life Science Products, Boston, MA, USA). Membranes were blocked and then incubated first with anti-phospho-protein antibody (p-IR Santa Cruz; Pser307 IRS1, p-Akt, and p-ACC Cell Signalling; p-ERK, BD Transduction Laboratories) and exposed to peroxidase-conjugated anti-mouse secondary antibodies. Immunoreactive bands were visualised by enhanced chemiluminescence (Western Lightning Chemiluminescence Reagent Plus, Perkin-Elmer, Boston, MA, USA) and exposure to autoradiograph film. The membranes were then stripped and reprobed with an antibody against the corresponding total protein (BD Transduction Laboratories, Cell Signalling or Santa Cruz) and then with $\beta$-actin (Sigma, St Louis, MO, USA) or HSP90 (BD Transduction Laboratories) to serve as an internal control for protein loading.

\section{mRNA Analyses}

Total RNA was prepared from frozen liver or from frozen subcutaneous adipose tissue using TRIpure isolation reagent (Roche). Hepatic mRNA expression levels of murine type I collagen- $\alpha-1$ (Col-I), transforming growth factor $\beta 1$ (TGF $\beta 1$ ), acyl-coenzyme A oxidase (ACO), cytochrome P450 2E1 (CYP2E1), CD36, aP2, PPAR $\gamma-1$ and phosphoenolpyruvate carboxykinase (PEPCK) were quantitated by realtime PCR. mRNA levels for CD36, aP2, PPAR $\gamma-1$, leptin and adiponectin were determined in adipose tissue. Primer pairs for transcripts of interest were designed using the Primer Express ${ }^{\mathrm{TM}}$ design software (Applied Biosystems). The ABI Prism 5700 PCR platform and SYBRgreen ${ }^{\circledR}$ mastermix were used for detection of the amplification product. The relative amount of mRNA was calculated by reference to a calibration curve and normalised to the level of expression of RPL19 mRNA, an invariant control.

\section{Statistical Analyses}

Results are presented as mean \pm standard deviation. The two-way ANOVA and Student's $t$-test were used to compare data from different treatment groups. When $P$ was less than 0.05 , differences were considered significant.

\section{Results}

\section{Pioglitazone Confers Protection against Steatohepatitis Induced by the MCD Diet}

Mice fed the MCD diet for 4 weeks developed prominent hepatic macrovesicular steatosis, conspicuous inflammation forming intralobular foci associated with increased hepatic lipid content and elevation of serum ALT levels (Figure 1c, Table 1). No or little hepatic fibrosis developed after 4 weeks MCD feeding as assessed on Sirius redstained liver sections (not shown). However, in those mice, hepatic type I collagen- $\alpha-1$, and TGF $\beta 1$ mRNA were significantly upregulated compared to control animals signing early fibrogenesis (Figure 1e and f).
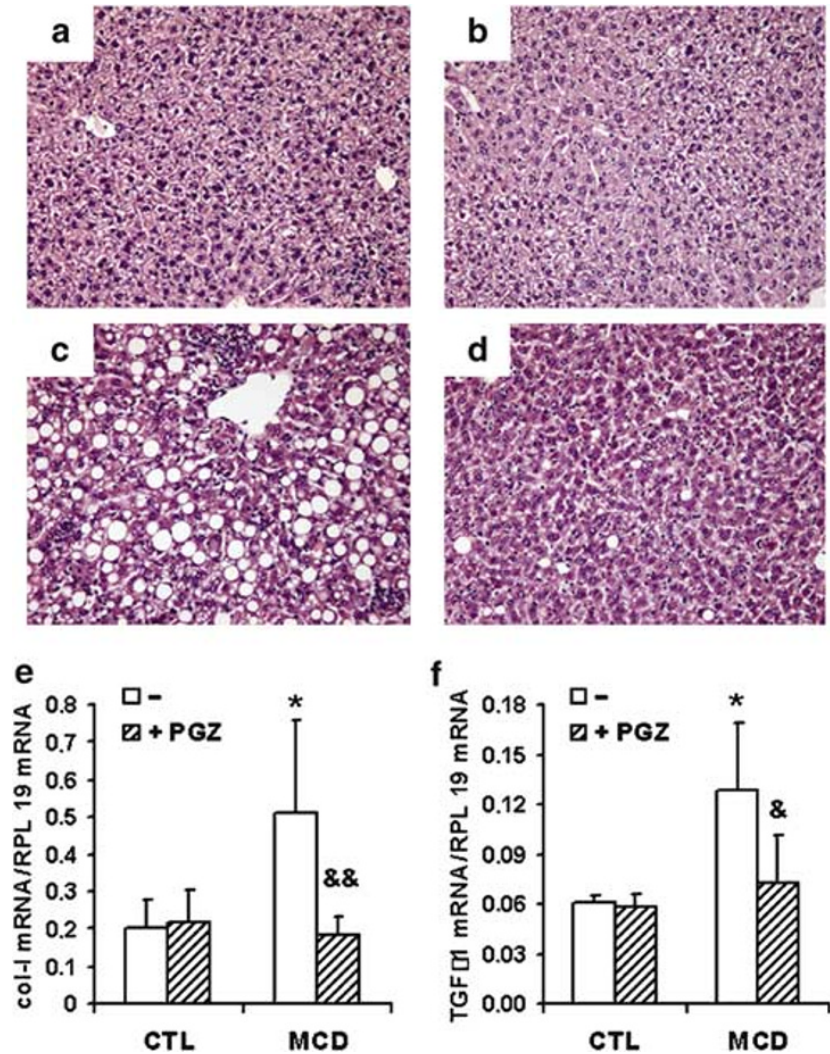

Figure 1 Effect of PGZ on steatohepatitis induced by the MCD diet. Representative liver sections from female C57BL6/J mice fed (a) the control diet, (b) the control diet together with PGZ treatment, (c) the MD diet or (d) the MCD diet together with PGZ treatment. Liver sections are stained with haematoxylin-eosin. The magnification is $\times 20$. (e) Hepatic collagen alpha1 type 1 mRNA expression and (f) hepatic TGF $\beta 1$ mRNA expression in the liver of control (CTL) or MCD-fed mice treated (hatched bars) or not (open bars) with PGZ. Values are standardised to the expression of RPL19 mRNA regarded as an invariant internal control and expressed as mean \pm s.d. for $n=5$ animals per group. ${ }^{*} P<0.05$ in MCD-fed $v s$ controls; ${ }^{*} P<0.05$ and $\& \& P<0.01$ in PGZ-treated, MCD-fed vs MCD-fed mice. 
PGZ significantly reduced the severity of hepatic steatosis and inflammation induced by the MCD diet (Figure 1d). It also significantly decreased intrahepatic lipid content, serum ALT levels and hepatic area occupied by inflammatory infiltrates (Table 1) compared to MCD-fed mice. In addition, PGZ treatment prevented the upregulation of hepatic collagen I and TGF $\beta 1$ mRNA expression seen after MCD feeding (Figure 1e and f).

\section{Effect of MCD Diet and PGZ on Peripheral Response to Insulin}

As previously described, mice fed the MCD diet for 4 weeks lost body weight (Table 2). Fasting blood glucose as well as fasting insulin levels were significantly lower in mice fed the MCD diet compared to controls, but serum leptin and adiponectin levels remained unaffected by the MCD diet (Table 2). After intraperitoneal glucose challenge, blood glucose levels increased less and were at all time lower in MCD-fed mice than in controls (Figure 2a) such as the area under the curve (AUC) of serum glucose was significantly lower in MCD compared to control-fed mice (Figure 2b). Intraperitoneal injection of insulin caused a more rapid and more pronounced reduction in blood glucose in MCDfed mice than in control mice (Figure 2c). These results indicate enhanced glucose tolerance and insulin hypersensitivity in MCD-fed mice, in accordance with observations by others. ${ }^{33}$

PGZ given together with the control or the MCD diet did not modify the body weight evolution (Table 2). However, consistent with its adipogenic

Table 1 Effect of MCD diet and PGZ on serum ALT, liver lipid content and hepatic inflammation

\begin{tabular}{lccc}
\hline Diet & $\begin{array}{c}\text { Serum ALT } \\
\text { (IU/l) }\end{array}$ & $\begin{array}{c}\text { Total hepatic lipids } \\
\text { (mg lipids/100g } \\
\text { liver) }\end{array}$ & $\begin{array}{c}\text { Inflammatory } \\
\text { area (\% of total } \\
\text { hepatic area) }\end{array}$ \\
\hline CTL & $19 \pm 4$ & $3.2 \pm 0.8$ & $0.003 \pm 0.002$ \\
CTL+PGZ & $22 \pm 5$ & $3.1 \pm 0.3$ & $0.009 \pm 0.005$ \\
MCD & $228 \pm 33^{\# \#}$ & $6.1 \pm 1.8^{\# \#}$ & $0.299 \pm 0.069^{\# \# \#}$ \\
MCD+PGZ & $169 \pm 18^{* *}$ & $4.6 \pm 1.3^{*}$ & $0.097 \pm 0.075^{* *}$
\end{tabular}

${ }^{\# \#} P<0.01$ and ${ }^{\# \# \# P} P<0.001$ in MCD compared to CTL; ${ }^{*} P<0.05$ and ${ }^{*} P<0.01$ in MCD+PGZ compared to MCD. action, ${ }^{2,35}$ PGZ significantly increased the mass of subcutaneous abdominal fat in both controls and MCD-fed mice (Table 2). Accordingly, PGZ treatment to both control and MCD-fed diet was associated with increased adipose expression of CD36 and adiponectin mRNA, two genes regulated by PPAR $\gamma$ (Table 3).

PGZ did not induce significant difference in fasting glucose, insulin or leptin levels in controls nor in MCD-fed mice, but PGZ increased serum adiponectin concentrations by a factor 2 in both dietary groups (Table 2). The systemic responses to insulin load were not altered by PGZ treatment in any of the dietary groups (Figure 2c). After glucose challenge, serum glucose levels decreased more rapidly in PGZ MCD-fed mice but not in PGZ control mice (Figure 2a and b).

\section{Effect of MCD Diet and PGZ on Intrahepatic Response to Insulin}

To assess the intrahepatic insulin sensitivity, we evaluated phosphorylation of intermediate proteins along the insulin-receptor signalling pathway in response to insulin in the various dietary groups.

There was no significant difference in the level of expression of the insulin receptor (IR) between CTL and MCD livers. In basal (unstimulated) conditions, little p-IR was detected whether in control or MCDfed livers. Insulin stimulation clearly increased the level of IR phosphorylation in CTL, but significantly less so in MCD livers (Figure 3). Akt protein expression was lower in MCD-fed than in control livers (Figure 4a and b). A bolus of insulin induced a marked increase in Akt phosphorylation in the liver of control mice (6.5-fold increase in the ratio of pAkt/Akt), but in MCD-fed mice, minimal phosphorylation of Akt occurred in response to insulin stimulus (1.5-fold over basal values; Figure 4c and d). Livers of control and MCD-fed mice had similar basal levels of ERK1/2 protein (Figure 5a), but as previously reported, ${ }^{28,32}$ basal phosphorylation of ERK1/2 is increased in MCD livers compared to controls (Figure 5a and b). Insulin significantly increased p-ERK1/2 in control livers but not in MCD livers (Figure 5c and d). Taken together, these data demonstrate that, despite systemic hypersensitivity to insulin, MCD-fed mice have hepatic resistance to

Table 2 Effects of MCD diet and PGZ on body weight, subcutaneous fat mass at biochemical markers

\begin{tabular}{lcccccc}
\hline Diet & Body weight (g) & $\begin{array}{c}\text { Subcutaneous } \\
\text { abdominal fat (g) }\end{array}$ & $\begin{array}{c}\text { Fasting glucose } \\
(\mathrm{mg} / \mathrm{dl})\end{array}$ & $\begin{array}{c}\text { Fasting insulin } \\
(\mathrm{ng} / \mathrm{ml})\end{array}$ & $\begin{array}{c}\text { Serum leptin } \\
(\mathrm{ng} / \mathrm{ml})\end{array}$ & $\begin{array}{c}\text { Serum adiponectin } \\
(\mu \mathrm{m} / \mathrm{ml})\end{array}$ \\
\hline CTL & $20.5 \pm 0.7$ & $0.24 \pm 0.05$ & $123 \pm 10$ & $2.1 \pm 0.4$ & $5.4 \pm 0.9$ & $7.2 \pm 1.2$ \\
CTL+PGZ & $20.6 \pm 0.3$ & $0.35 \pm 0.05^{* *}$ & $109 \pm 7$ & $1.8 \pm 0.2$ & $6.7 \pm 1.9$ & $14.1 \pm 3.4^{* *}$ \\
MCD & $12.1 \pm 0.4^{\# \# \#}$ & $0.09 \pm 0.01^{\# \# \#}$ & $91 \pm 16^{\# \#}$ & $1.2 \pm 0.5^{\# \#}$ & $4.8 \pm 1.1$ & $9.0 \pm 2.4$ \\
MCD+PGZ & $12.2 \pm 0.4^{\# \# \#}$ & $0.17 \pm 0.05^{*}$ & $82 \pm 11^{\# \#}$ & $1.1 \pm 0.3$ & $6.2 \pm 1.5$ & $17.6 \pm 3.1^{* *}$ \\
\hline
\end{tabular}

${ }^{\# \# P} P 0.01$ and ${ }^{\# \#} P<0.001$ in MCD compared to CTL controls. ${ }^{*} P<0.05$ and ${ }^{*} P<0.01$ in PGZ-treated vs mice fed the corresponding diet. 
60
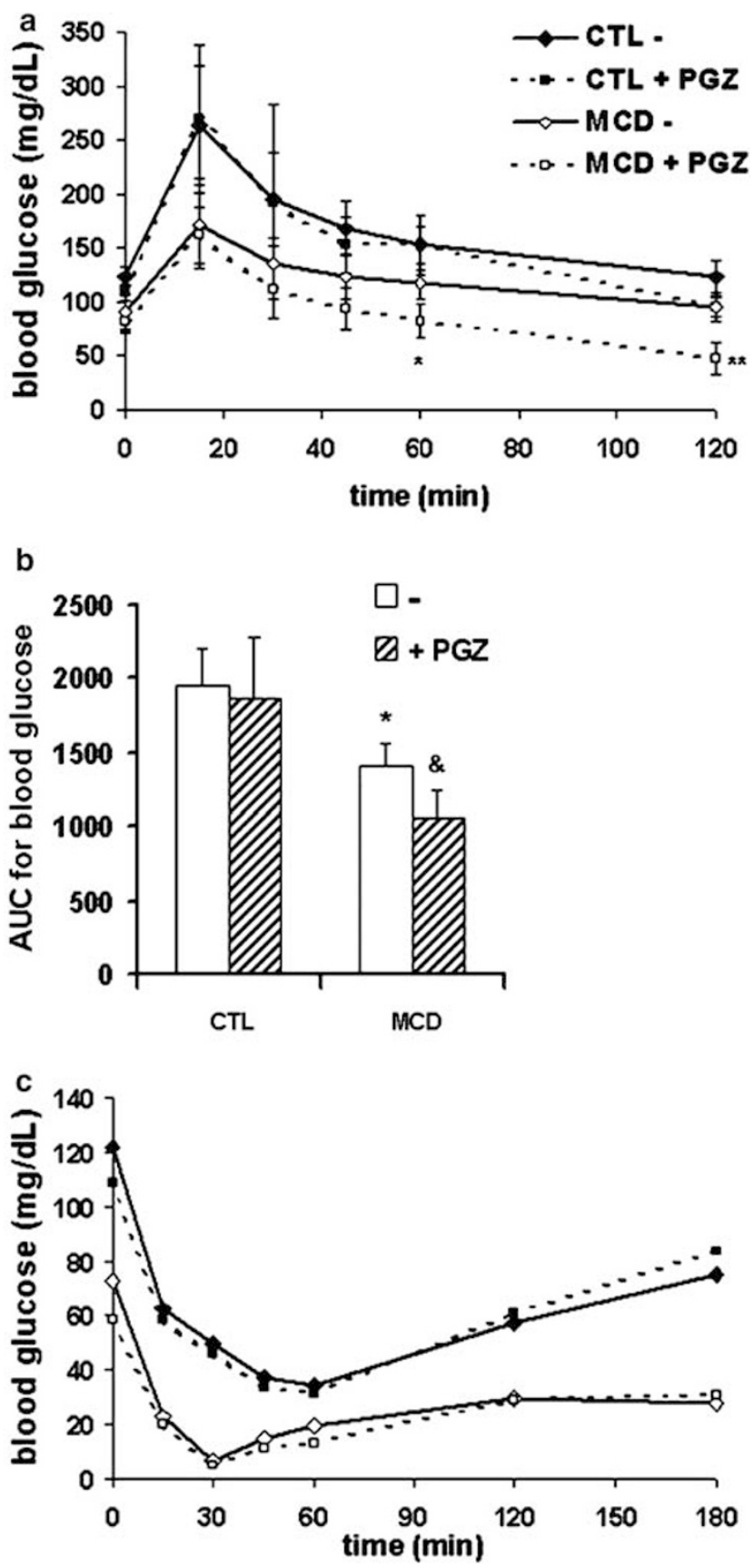

Figure 2 Effect of MCD diet and PGZ on blood glucose levels after intraperitoneal glucose or insulin challenge. (a) Curves of blood glucose concentrations after intraperitoneal glucose challenge in CTL (black diamond, continuous line), MCD-fed (open diamond, continuous line), PGZ-treated CTLl (black square, dotted line) and PGZ-treated MCD-fed (open square, dotted line) mice. (b) Graph representing the AUC of blood glucose after intraperitonea glucose challenge in CTL and MCD-fed mice with (hatched bars) or without (open bars) PGZ treatment. (c) Curves for blood glucose concentrations after intraperitoneal insulin challenge. Values are mean \pm s.d. for $n=5$ animals per group. ${ }^{*} P<0.05$ in MCD-fed vs controls; ${ }^{\circledR} P<0.05$ in PGZ-treated MCD-fed vs MCD-fed mice.

insulin action. Blockade of insulin signalling occurs at the earliest event of the cascade (the phosphorylation of the receptor) and leads to resistance on
Table 3 CD36 and adiponectin mRNA expression in adipose tissue

\begin{tabular}{lcc}
\hline Diet & CD 36 mRNA & Adiponectin $m R N A$ \\
\hline CTL & $1 \pm 0.4$ & $1 \pm 0.27$ \\
CTL+PGZ & $3 \pm 0.8^{*}$ & $1.65 \pm 0.17^{* *}$ \\
MCD & $2.3 \pm 0.4^{\# \#}$ & $1.29 \pm 0.37$ \\
MCD+PGZ & $4 \pm 0.4^{*}$ & $1.75 \pm 0.32^{*}$ \\
\hline
\end{tabular}

Data are arbitrary units \pm s.d. for $n=5$ per group. Data represent the ratio of mRNA expression of the gene of interest to that of RPL19, regarded as invariant (internal) control. ${ }^{\# \#} P<0.01$ in MCD compared to CTL. ${ }^{*} P<0.05$ and ${ }^{*} P<0.01$ in PGZ-treated $v s$ mice fed the corresponding diet.

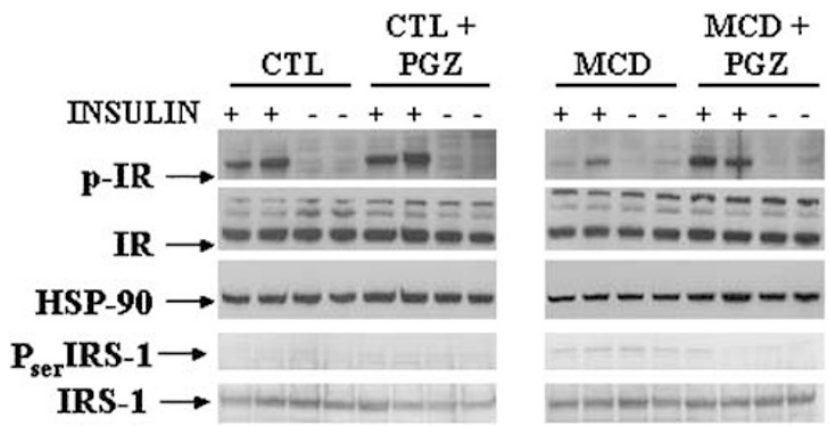

Figure 3 Effect of MCD and PGZ treatment on the phosphorylation of the IR in response to insulin injection and on serine phosphorylation of IRS1. Representative Western blot of p-IR, IR, Pser307 IRS1 and IRS1 proteins in the liver from CTL and MCDfed mice with or without PGZ treatment. Blots were reprobed with HSP-90 to assess equal loading.

both the metabolic pathway governed by Akt and the mitogenic pathway controlled by ERK1/2.

In control mice, PGZ treatment did not alter basal hepatic expression of IR and Akt, nor their phosphorylation in response to insulin (Figures 3 and 4). In MCD-fed mice, PGZ treatment increased the basal expression of Akt protein to levels equal or higher to those observed in control-fed mice (Figure 4a and b). In addition, PGZ dramatically increased the phosphorylation of both IR and Akt in response to insulin stimulus, restoring to normal the response that was almost abolished in MCD-fed mice (Figures 3 and $4 \mathrm{c}$ and $\mathrm{d}$ ).

In controls, PGZ did not significantly modify the hepatic expression of ERK1/2 proteins, the basal level of pERK1/2 or the magnitude of the response to insulin stimulus. In MCD livers, PGZ treatment was associated with a significant increase in the basal level of p-ERK1/2, this level of phosphorylation was not further enhanced by insulin (Figure 5). Thus, contrasting with the restoration of IR and Aktphosphorylation in response to insulin induced by PGZ treatment to MCD-fed mice, PGZ was not associated with a restoration of the activation of the ERK pathway by insulin. Alternatively, as PGZ treatment is associated with a significant induction of p-ERK, additional stimulation through the IR cannot be further recruited. 

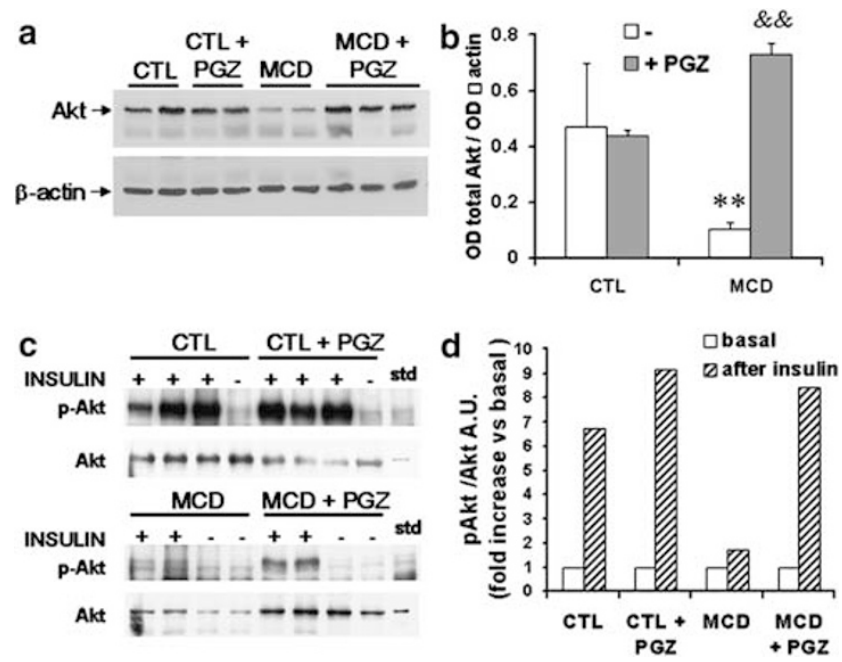

Figure 4 Akt phosphorylation in response to insulin is impaired in the liver of MCD-fed mice and restored by PGZ treatment. (a) Representative Western blot of Akt protein expression in the liver from CTL and MCD-fed mice with or without PGZ treatment. Blots were reprobed with $\beta$-actin to assess equal loading. (b) Densitometric values for Akt protein, at basal level, among the different groups. (c) Western blot of hepatic p-Akt and Akt after insulin stimulus (+) or PBS (-) in CTL and MCD-fed mice with or without PGZ treatment. (d) Graph representing the phosphorylation of Akt in response to insulin. Data are the ratio of densitometric values for p-Akt on Akt expressed as fold increase compared to basal $(-)$ levels $(n=3$ per group and treatment). ${ }^{* *} P<0.01$ in MCD-fed $v s$ controls; ${ }^{\& \&} P<0.01$ in PGZ-treated MCDfed vs MCD-fed mice and vs PGZ-treated control mice.

Serine phosphorylation of IRS proteins has been shown to inhibit insulin-mediated signalling. The level of expression of IRS1 (Figure 3) and IRS2 (not shown) does not significantly differ among groups. Serine phosphorylation of IRS1 was barely detectable in CTL livers, appeared more readily in MCD livers and was slightly decreased upon PGZ treatment. Insulin stimulus did not induce apparent changes in the level of phosphoserine-IRS1. This suggests that inhibitory phosphorylation of IRS1 may be a mechanism, in addition to the defect in IR phosphorylation, that plays a role in intrahepatic insulin resistance in MCD livers. Studies from Czaja's group have suggested that impaired intrahepatic insulin signalling in MCD-fed mice might be the consequence of increased CYP2E1-generated oxidative stress. ${ }^{34}$ We therefore analysed expression of CYP2E1 mRNA and found expectedly that MCD diet induced CYP2E1 mRNA,${ }^{27}$ and that this induction was dampened by PGZ treatment to MCD-fed mice (Table 4).

Inhibition of phosphoenolpyruvate carboxykinase (PEPCK), a rate-limiting enzyme in gluconeogenesis, is one of the processes by which insulin controls hepatic glucose output. PEPCK mRNA was expressed at similar levels in CTL and MCD livers. PGZ treatment significantly reduced PEPCK expression in MCD but not in control mice (Figure 6), while it did not alter circulating insulin levels (Table 2). In line with the effect of PGZ on activation

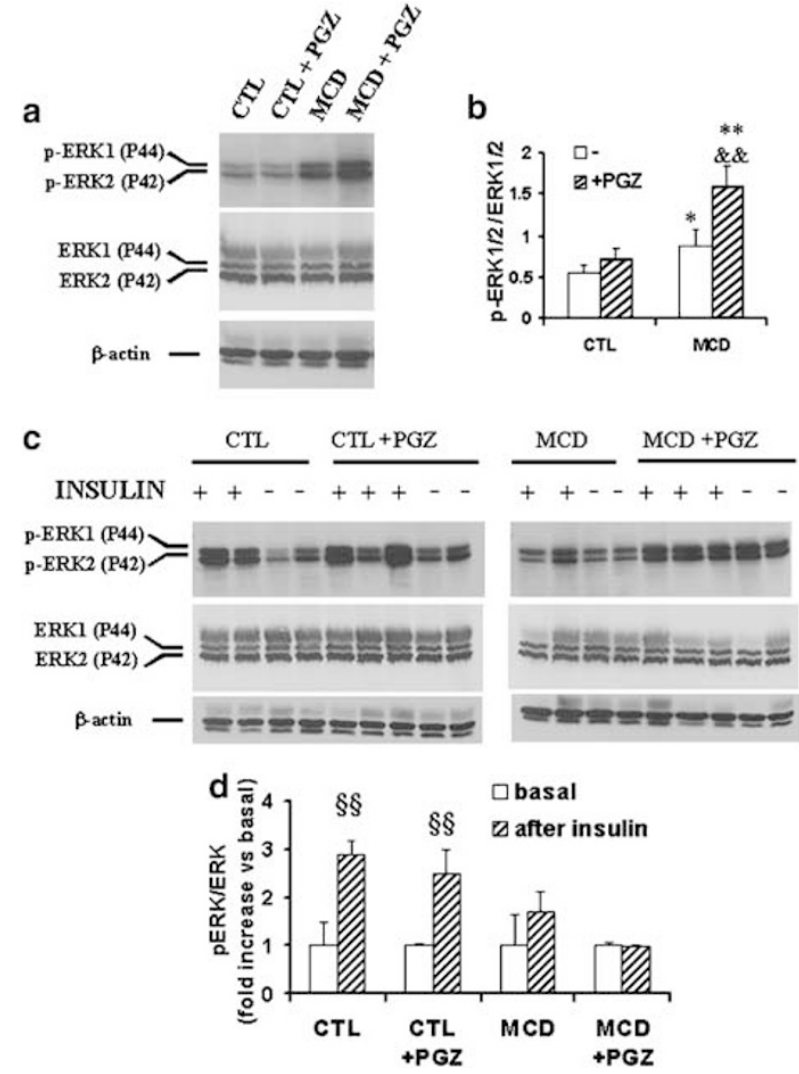

Figure 5 Effect of MCD diet and PGZ treatment of ERK1/2 phosphorylation in response to insulin stimulus. (a) Representative Western blot of p-ERK1/2 and ERK1/2 protein expression in the liver from CTL and MCD-fed mice with or without PGZ treatment. Blots were reprobed with $\beta$-actin to assess equal loading. (b) Densitometric values for $P$-ERK1/2 protein, at basal level, among the different groups, expressed as the ratio of pERK1/2 to ERK1/2. Data are mean \pm s.d. for $n=3$ per group. (c) Western blot of hepatic p-ERK1/2, ERK1/2 and $\beta$-actin after insulin stimulus $(+)$ or PBS (-) in CTL and MCD-fed mice with or without PGZ treatment. (d) Graph representing the phosphorylation of ERK1/2 in response to insulin. Data are the ratio of densitometric values for p-ERK on ERK expressed as fold increase compared to basal $(-)$ levels $(n=3$ per group and treatment). ${ }^{*} P<0.05,{ }^{*} P<0.01$ vs controls; ${ }^{\star} P P<0.01$ in PGZ-treated MCDfed vs MCD-fed mice. ${ }^{\$ s} P>0.01$ in insulin stimulated $v s$ basal.

of insulin signalling molecules, downregulation of PEPCK suggests increased sensitivity to insulin in PGZ-MCD livers.

\section{Effects of PGZ on $\beta$-Oxidation and Lipid Storage}

As mentioned above, PGZ treatment inhibits hepatic steatosis caused by the MCD diet (Figure 1 and Table 1). Hepatic ACO codes for the rate-limiting enzyme of fatty acids $\beta$-oxidation. ACO was reduced in the livers of MCD-fed mice compared to controls compatible with the proposal that reduced combustion of fatty acids participate to steatosis induced by the MCD diet. ${ }^{29,30}$ PGZ treatment to MCD-fed mice did not modify the expression of ACO (Table 4). ACC regulates $\beta$-oxidation by controlling the levels 
Table 4 Effects of MCD diet and PGZ treatment on hepatic CYP2E1, PPAR $\gamma$, CD36 and ACO mRNA

\begin{tabular}{lcllc}
\hline Diet & CYP2E1 mRNA & PPAR -1 mRNA & CD 36 mRNA & ACO mRNA \\
\hline CTL & $1 \pm 0.08$ & $0.25 \pm 0.01$ & $0.03 \pm 0.01$ & $1.6 \pm 0.2$ \\
CTL+PGZ & $1.47 \pm 0.87$ & $0.28 \pm 0.04$ & $0.04 \pm 0.02$ & ND \\
MCD & $2.47 \pm 0.82^{\#}$ & $1.22 \pm 0.14^{\# \#}$ & $0.09 \pm 0.02^{\# \#}$ & $0.9 \pm 0.4^{\#}$ \\
MCD+PGZ & $1.43 \pm 0.047^{*}$ & $1.09 \pm 0.12$ & $0.15 \pm 0.02^{*}$ & $0.6 \pm 0.2$ \\
\hline
\end{tabular}

Data are arbitrary units \pm s.d. for $n=5$ per group. Data represent the ratio of mRNA expression of the gene of interest to that of RPL19, regarded as invariant (internal) control. ${ }^{\#} P<0.05$ and ${ }^{\# \#} P<0.01$ in MCD compared to CTL. ${ }^{*} P<0.05$ in PGZ-treated vs mice fed the corresponding diet. ND: not determined.

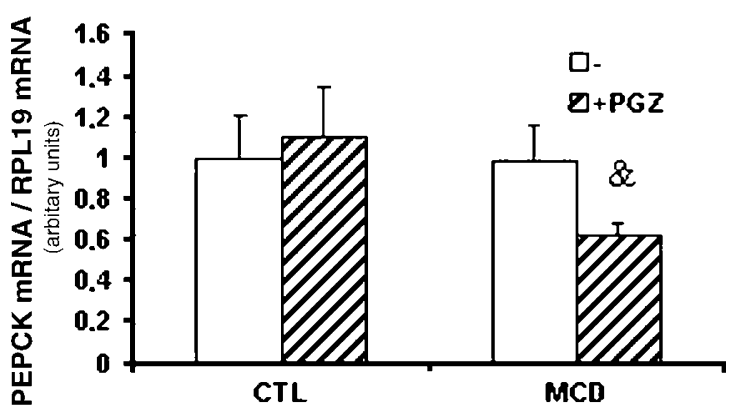

Figure 6 Effect of MCD diet and PGZ treatment on phosphoenopyruvate carboxykinase (PEPCK) gene expression. Hepatic PEPCK mRNA expression in the liver of control (CTL) or MCD-fed mice treated (hatched bars) or not (open bars) with PGZ. Values are standardised to the expression of RPL19 mRNA regarded as an invariant internal control and expressed as mean \pm s.d. for $n=5$ animals per group. ${ }^{\circledR} P<0.05$ in PGZ-treated, MCD-fed vs MCD-fed mice.

of malonyl-coA and thereby the entrance of fatty acids into the mitochondria. MCD was associated with a $50 \%$ increase in the pACC/ACC ratio (Figure 7a). However, because the ACC content was decreased in those livers (Figure 7b), the total amount of pACC was similar to that found in the liver of control mice, and unaltered by PGZ treatment (Figure 7c). Also, hepatic mRNA expression of PPAR $\gamma 1$ - and PPAR $\gamma$-regulated fatty acid transporter CD36 were increased in MCD livers (Table 4). While treatment with PPAR $\gamma$ ligand PGZ did not alter PPAR $\gamma$ mRNA expression, it induced CD36 expression in MCD livers (Table 4). Therefore, although MCD diet might favour steatosis by decreasing $\beta$ oxidation (as suggested by decreased ACO) and increased fatty acid uptake (resulting from increased CD36 expression), reduction of steatosis observed under PGZ treatment might not be explained by a stimulation of $\beta$-oxidation, or a limitation of fatty acid transport into the hepatocytes.

\section{Discussion}

In humans, NASH occurs in association with insulin resistance and obesity. While it is accepted that hepatic fat accumulation and insulin resistance are linked, the mechanism for this relationship remains unclear. Mice fed a lipogenic diet deficient
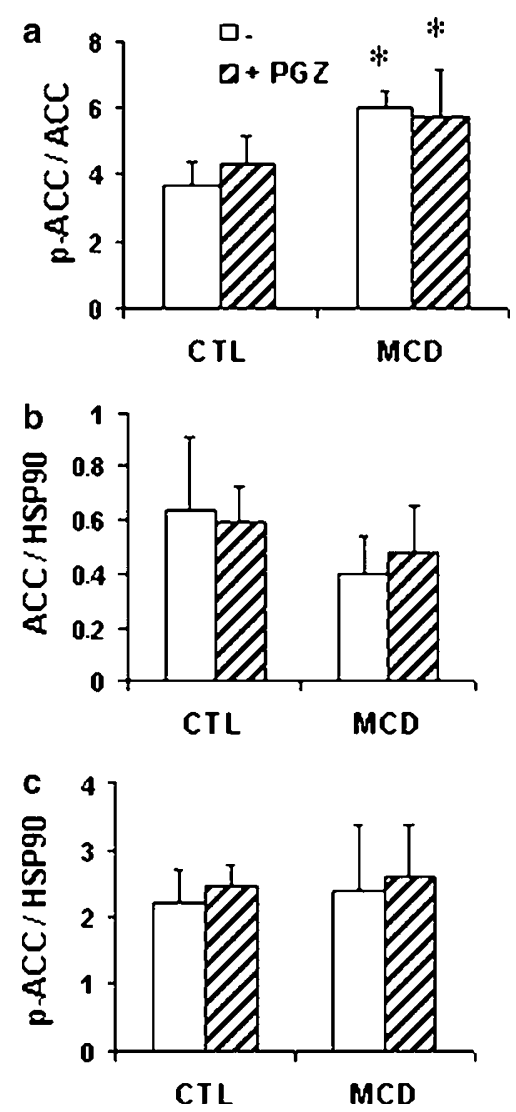

Figure 7 Effect of MCD diet and PGZ treatment on expression and phosphorylation of ACC. Graph representing the ratio of densitometric values of Western blots for (a) pACC on ACC, (b) ACC on HSP90 regarded as an invariant internal control for equality of loading, and (c) pACC/HSP90. Data are mean \pm s.d. for $n=5$ animals per group. ${ }^{*} P<0.05$ in MCD-fed vs corresponding CTL mice.

in methionine and choline are used as a model of steatohepatitis. Although those mice lose weight and have normal or hyper-normal systemic sensitivity to insulin, they develop intrahepatic insulin resistance. Our data demonstrate that PGZ prevents hepatic fat accumulation and steatohepatitis and also prevents intrahepatic insulin resistance in MCD-fed mice.

Choline and methionine deficiency induces steatosis as a result of both decreased availability in phosphatidylcholine, which leads to membrane 
dysfunction and impaired excretion of VLDL, and altered regulation of genes controlling glucose and lipid metabolism such as ACO, the rate-limiting enzyme for $\beta$-oxidation. ${ }^{29,36}$ PGZ induces the production of adiponectin. ${ }^{37}$ In the liver, adiponectin activates AMP kinase (AMPK) and PPAR $\alpha$ leading to increased fatty acid combustion and decreased hepatic lipid content. ${ }^{38}$ In our model, despite a twofold increase in serum adiponectin concentrations, phosphorylation of ACC (a substrate for AMPK) or PPAR- $\alpha$-dependent expression of ACO were not significantly altered. This suggests that the antisteatotic effect of PGZ is not related to stimulation of hepatic lipid burning.

PPAR $\gamma$ agonist PGZ promotes fatty acid storage and uptake in adipose tissue. ${ }^{7,35}$ Accordingly, the mass of subcutaneous abdominal adipose tissue increased in PGZ-treated animals. Therefore, diversion of fat from the liver to the adipocytes may participate to the reduction of steatosis by PGZ. ${ }^{2,5}$ Beside adipocytes, PPAR $\gamma$ is also able to induce an adipogenic transformation of other cell types, and, in particular, hepatocytes. ${ }^{39}$ Enhanced expression of PPAR $\gamma$ and PPAR $\gamma$-regulated fatty acid transporter CD36 in the liver of MCD-fed mice suggests that $\operatorname{PPAR} \gamma$ may exert an adipogenic effect favouring steatosis. However, while PGZ further induces CD36 expression in MCD livers, this was associated with a dramatic reduction of steatosis. This implies that PPAR $\gamma$-mediated adipogenic transformation of hepatocytes has no physiological significance in this model, or, that this effect is overpowered by another antisteatotic action of PGZ.

Thus, increased intrahepatic lipid burning does not appear to be the process by which PGZ reduces steatosis in this model. Rather, the increased fat mass suggests that decreased steatosis may be the consequence of diversion of fat storage from the liver to the adipose tissue promoted by direct stimulation of PPAR $\gamma$ in adipocytes.

Contrasting with the metabolic status in patients with NASH, MCD-fed mice lose weight, are depleted from peripheral fat, have low fasting glucose and insulin serum levels, as well as normal leptin and adiponectin levels. In accordance with others, our metabolic studies demonstrate that their tolerance to glucose and their systemic insulin sensitivity are increased,,$^{33}$ an observation that has also been made in calorically restricted animals. ${ }^{40,41}$ Despite this, and by contrast to calorie restriction, MCD-fed mice developed significant intrahepatic insulin resistance. The activation, by insulin, of both the mitogenic (ERK) and the metabolic (Akt) pathways controlling gluconeogenesis, glycogenolysis, glycogen synthesis ${ }^{42}$ are blunted in MCD-fed livers. Defective phosphorylation of the insulin receptor appears as an important mechanism for insulin resistance in this model, although additional blockade along the pathway may also result from increased serine (inhibitory) phosphorylation of IRS1. Treatment of MCD-fed mice with PGZ improved intrahepatic insulin resistance as demonstrated by the restoration of normal phosphorylation of IR and Akt in response to insulin stimulus, together with apparent decrease in Pserine-IRS1. Studies have documented that inappropriate regulation of glucose production by the liver is an important component of the diabetic states, and that TZD drugs exert their insulin-sensitising effect by potentiation of insulin-mediated activation of Akt. ${ }^{43}$ In MCD-fed mice, impaired intrahepatic insulin resistance and defective activation of Akt were not associated with altered expression of PEPCK, a rate-limiting enzyme in gluconeogenesis nor with decreased glucose-lowering effect of insulin in vivo. TZD treatment had remarkable effect on intrahepatic insulin signalling and downregulation of PEPCK. In turn, this could explain, via better control of insulin over hepatic glucose output, the faster decrease of blood glucose levels after glucose challenge in TZD-treated MCD mice. However, and contrasting with this proposition, the treatment had no significant consequence on in vivo response to insulin. As systemic insulin sensitivity is clearly enhanced, increased glucose uptake by peripheral tissue may compensate for increased hepatic glucose output expected from intrahepatic insulin resistance in MCD-fed mice. Alternatively, hepatic glucose production might be limited in these animals as a consequence of cachexia and depleted hepatic glycogen stores. Hyperinsulinemic clamp studies and analysis of muscle and adipose insulin signalling are needed for further understanding.

Whether, under PGZ treatment, amelioration of intrahepatic insulin resistance is the cause or the consequence of improved peripheral insulin sensitivity has been debated., ${ }^{4,6,8}$ In our setting, systemic and intrahepatic insulin resistances are clearly dissociated and correction of intrahepatic insulin resistance by PGZ is independent on systemic insulin sensitisation. Adiponectin-dependent control of hepatic glucose production appears as a critical mediator of PPAR $\gamma$-agonist-mediated improvement of insulin sensitivity. ${ }^{6,44,45}$ The two-fold increase in serum adiponectin concentrations induced by PGZ treatment was associated with decreased expression of PEPCK and restoration of intrahepatic insulin sensitivity in MCD-fed livers; while a similar increase in adiponectin in control mice does not significantly affect insulin-dependent signalling cascade. On the other hand, accumulation of intracellular fatty-acid metabolites and hepatic steatosis may lead to hepatic insulin resistance, by direct interference with intermediate effectors of intracellular IR-dependent signalling pathways. ${ }^{24,26}$ Such alteration may also be produced by reactive oxygen species generated by CYP2E1, whose expression is induced in the liver of MCD-fed mice. ${ }^{27,34}$ PGZ treatment to MCD-fed mice resulted in the clearance of steatosis, decreased induction of CYP2E1 and improvement of intrahepatic insulin signalling. Increase in lipid metabolites (which are 
substrates for CYP2E1) has been proposed as a plausible mechanism for CYP2E1 induction in MCD livers. ${ }^{27,29}$ To the best of our knowledge, the participation of PPAR $\gamma$ in CYP2E1 gene regulation has never been explored. Because PGZ does not influence the level of CYP2E1 expression in normal livers, prevention of CYP2E1 upregulation by PGZ in MCD livers is more likely linked to decreased fat. Therefore, abolition of CYP2E1 induction might mechanistically participate to the restoration of intrahepatic insulin sensitivity associated with steatosis clearance induced by PPAR $\gamma$ agonists in this model. Experiments using adiponectin KO mice might be helpful in determining the respective roles of adiponectin and decreased steatosis by preferential storage of fat in the adipose tissue in the hepatic insulin-sensitising effect of PGZ in MCD-induced steatohepatitis.

Finally, PGZ reduced hepatic inflammation and early fibrogenesis in MCD-fed mice. As demonstrated elsewhere, clearance of hepatic lipids is sufficient to prevent or revert steatohepatitis. ${ }^{29,30}$ Additionally, modulation of inflammation by PPAR $\gamma$ itself or PPAR $\gamma$-regulated adipocytokines might be implicated. ${ }^{46-49}$ Additional studies using mice null for adipocytokines or their receptors or in which PPAR $\gamma$ will be conditionally and tissue specifically knocked-down would be required to delineate the specific roles of adipose tissue, liver and inflammatory cells in decreased inflammation obtained by PPAR $\gamma$ agonist treatment. PGZ, as described in several other studies, ${ }^{50}$ increases the activation of the MAPK/ERK pathway, and particularly so in MCD livers. Because MAPKs are well-known growth regulators, it will be interesting to test in further studies whether PGZ-induced activation of this pathway stimulates the replacement of damaged hepatocytes and thereby decreases noxious consequences of steatohepatitis.

In conclusion, in the MCD diet model of steatohepatitis, there is dissociation between enhanced systemic insulin sensitivity and intrahepatic resistance to insulin. PGZ treatment to MCD-fed mice alleviates steatosis and steatohepatitis. This effect was associated with improved intrahepatic insulin sensitivity. Our data support the proposition that reduced steatosis under PGZ treatment is at least partly the consequence of diversion of fat from liver to adipose storage. Reduced steatosis, prevention of CYP2E1 induction and increased serum adiponectin might mechanistically participate to the restoration of intrahepatic insulin sensitivity in PGZ-treated MCD-fed mice.

\section{Acknowledgement}

We thank Dr Christine Sempoux (Pathology Unit, Cliniques Universitaires St-Luc, Université Catholique de Louvain, Brussels, Belgium) for her contribution in the histopathological evaluation of liver damage in this study. This work was supported by a grant from the Belgian 'Fonds National de la recherche Scientifique' (FNRS convention no: 3.4507.04) and by Grant 05/10-328 (action de recherche concertée de la direction de la recherché scientifique de la communauté française de Belgique). IL is research associated with the FNRS. Authors have no duality of interest to declare.

\section{References}

1 Berger J, Moller DE. The mechanisms of action of PPARs. Annu Rev Med 2002;53:409-435.

2 Yki-Jarvinen H. Thiazolidinediones. N Engl J Med 2004;351:1106-1118.

3 Gerhold DL, Liu F, Jiang G, et al. Gene expression profile of adipocyte differentiation and its regulation by peroxisome proliferator-activated receptor-gamma agonists. Endocrinology 2002;143:2106-2118.

4 Olefsky JM. Treatment of insulin resistance with peroxisome proliferator-activated receptor gamma agonists. J Clin Invest 2000;106:467-472.

5 Evans RM, Barish GD, Wang YX. PPARs and the complex journey to obesity. Nat Med 2004;10:355-361.

6 Bouskila M, Pajvani UB, Scherer PE. Adiponectin: a relevant player in PPARgamma-agonist-mediated improvements in hepatic insulin sensitivity? Int J Obes (Lond) 2005;29(Suppl 1):S17-S23.

7 Okuno A, Tamemoto $\mathrm{H}$, Tobe $\mathrm{K}$, et al. Troglitazone increases the number of small adipocytes without the change of white adipose tissue mass in obese Zucker rats. J Clin Invest 1998;101:1354-1361.

8 Lee CH, Olson P, Evans RM. Minireview: lipid metabolism, metabolic diseases, and peroxisome proliferator-activated receptors. Endocrinology 2003;144: 2201-2207.

9 Yamauchi T, Kamon J, Waki H, et al. The fat-derived hormone adiponectin reverses insulin resistance associated with both lipoatrophy and obesity. Nat Med 2001;7:941-946.

10 Gavrilova O, Haluzik M, Matsusue K, et al. Liver peroxisome proliferator-activated receptor gamma contributes to hepatic steatosis, triglyceride clearance, and regulation of body fat mass. J Biol Chem 2003;278: 34268-34276.

11 Way JM, Harrington WW, Brown KK, et al. Comprehensive messenger ribonucleic acid profiling reveals that peroxisome proliferator-activated receptor gamma activation has coordinate effects on gene expression in multiple insulin-sensitive tissues. Endocrinology 2001; 142:1269-1277.

12 Hevener AL, He W, Barak Y, et al. Muscle-specific $\operatorname{PPAR} \gamma$ deletion causes insulin resistance. Nat Med 2003;9:1491-1497.

13 Norris AW, Chen L, Fisher SJ, et al. Muscle-specific PPARgamma-deficient mice develop increased adiposity and insulin resistance but respond to thiazolidinediones. J Clin Invest 2003;112:608-618.

14 Galli A, Crabb DW, Ceni E, et al. Antidiabetic thiazolidinediones inhibit collagen synthesis and hepatic stellate cell activation in vivo and in vitro. Gastroenterology 2002;122:1924-1940.

15 Dubois M, Pattou F, Kerr-Conte J, et al. Expression of peroxisome proliferator-activated receptor gamma 
(PPARgamma) in normal human pancreatic islet cells. Diabetologia 2000;43:1165-1169.

16 Shashkin P, Dragulev B, Ley K. Macrophage differentiation to foam cells. Curr Pharm Des 2005;11: 3061-3072.

17 Desreumaux P, Dubuquoy L, Nutten S, et al. Attenuation of colon inflammation through activators of the retinoid X receptor (RXR)/peroxisome proliferatoractivated receptor gamma (PPARgamma) heterodimer. A basis for new therapeutic strategies. J Exp Med 2001; 193:827-838.

18 McCullough A. The Epidemilogy and Risk Factors of NASH. Fatty Liver Disease: NASH and Related Disorders (Chapter 3). Blackwell Publishers: Oxford, UK, 2004, pp 23-37.

19 Brunt EM. Pathology of nonalcoholic steatohepatitis. Hepatol Res 2005;33:68-71.

20 Bugianesi E, McCullough AJ, Marchesini G. Insulin resistance: a metabolic pathway to chronic liver disease. Hepatology 2005;42:987-1000.

21 Shulman GI. Cellular mechanisms of insulin resistance. J Clin Invest 2000;106:171-176.

22 Marchesini G, Brizi M, Morselli-Labate AM, et al. Association of nonalcoholic fatty liver disease with insulin resistance. Am J Med 1999;107:450-455.

23 Sanyal AJ, Campbell-Sargent C, Mirshahi F, et al. Nonalcoholic steatohepatitis: association of insulin resistance and mitochondrial abnormalities. Gastroenterology 2001;120:1183-1192.

24 Kim JK, Fillmore JJ, Chen Y, et al. Tissue-specific overexpression of lipoprotein lipase causes tissuespecific insulin resistance. Proc Natl Acad Sci USA 2001;98:7522-7527.

25 Kraegen EW, Clark PW, Jenkins AB. Development of muscle insulin resistance after liver insulin resistance in high-fat-fed rats. Diabetes 1991;40:1397-1403.

26 Samuel VT, Liu ZX, Qu X, et al. Mechanism of hepatic insulin resistance in non-alcoholic fatty liver disease. J Biol Chem 2004;279:32345-32353.

27 Leclercq IA, Farrell GC, Field J, et al. CYP2E1 and CYP4A as microsomal catalysts of lipid peroxides in murine nonalcoholic steatohepatitis. J Clin Invest 2000;105:1067-1075.

28 Leclercq IA, Farrell GC, Sempoux C, et al. Curcumin inhibits NF-kappaB activation and reduces the severity of experimental steatohepatitis in mice. J Hepatol 2004;41:926-934.

29 Ip E, Farrell GC, Robertson G, et al. Central role of PPARalpha-dependent hepatic lipid turnover in dietary steatohepatitis in mice. Hepatology 2003;38: 123-132.

30 Ip E, Farrell G, Hall P, et al. Administration of the potent PPARalpha agonist, $\mathrm{Wy}-14,643$, reverses nutritional fibrosis and steatohepatitis in mice. Hepatology 2004;39:1286-1296.

31 dela PA, Leclercq I, Field J, et al. NF-kappaB activation, rather than TNF, mediates hepatic inflammation in a murine dietary model of steatohepatitis. Gastroenterology 2005;129:1663-1674.

32 Schattenberg JM, Singh R, Wang Y, et al. JNK1 but not JNK2 promotes the development of steatohepatitis in mice. Hepatology 2006;43:163-172.

33 Rinella ME, Green RM. The methionine-choline deficient dietary model of steatohepatitis does not exhibit insulin resistance. J Hepatol 2004;40:47-51.

34 Schattenberg JM, Wang Y, Singh R, et al. Hepatocyte CYP2E1 overexpression and steatohepatitis lead to impaired hepatic insulin signaling. J Biol Chem 2005; 280:9887-9894

35 Yamauchi T, Kamon J, Waki H, et al. The mechanisms by which both heterozygous peroxisome proliferatoractivated receptor gamma (PPARgamma) deficiency and PPARgamma agonist improve insulin resistance. J Biol Chem 2001;276:41245-41254.

36 Farrell GC. Animal Models of Steatohepatitis. Fatty Liver Disease: NASH and Related Disorders (Chapter 8). Blackwell Publishers: Oxford, UK, 2004, pp 91-108.

37 Iwaki M, Matsuda M, Maeda N, et al. Induction of adiponectin, a fat-derived antidiabetic and antiatherogenic factor, by nuclear receptors. Diabetes 2003;52: 1655-1663.

38 Kadowaki T, Yamauchi T. Adiponectin and adiponectin receptors. Endocr Rev 2005;26:439-451.

$39 \mathrm{Yu} \mathrm{S}$, Matsusue K, Kashireddy P, et al. Adipocytespecific gene expression and adipogenic steatosis in the mouse liver due to peroxisome proliferator-activated receptor gamma1 (PPARgamma1) overexpression. J Biol Chem 2003;278:498-505.

40 Bordone L, Guarente L. Calorie restriction, SIRT1 and metabolism: understanding longevity. Nat Rev Mol Cell Biol 2005;6:298-305.

41 Masternak MM, Al Regaiey KA, Rosario Lim MM, et al. Effects of caloric restriction on insulin pathway gene expression in the skeletal muscle and liver of normal and long-lived GHR-KO mice. Exp Gerontol 2005;40: 679-684.

42 Cho $\mathrm{H}, \mathrm{Mu}$ J, Kim JK, et al. Insulin resistance and a diabetes mellitus-like syndrome in mice lacking the protein kinase Akt2 (PKB beta). Science 2001;292: 1728-1731.

43 Jiang G, Dallas-Yang Q, Li Z, et al. Potentiation of insulin signaling in tissues of Zucker obese rats after acute and long-term treatment with PPARgamma agonists. Diabetes 2002;51:2412-2419.

44 Combs TP, Wagner JA, Berger J, et al. Induction of adipocyte complement-related protein of 30 kilodaltons by PPARgamma agonists: a potential mechanism of insulin sensitization. Endocrinology 2002;143 998-1007.

45 Kubota N, Terauchi Y, Kubota T, et al. Pioglitazone ameliorates insulin resistance and diabetes by both adiponectin-dependent and -independent pathways. J Biol Chem 2006;281:8748-8755.

46 Delerive P, Fruchart JC, Staels B. Peroxisome proliferator-activated receptors in inflammation control. J Endocrinol 2001;169:453-459.

47 Yokota T, Oritani K, Takahashi I, et al. Adiponectin, a new member of the family of soluble defense collagens, negatively regulates the growth of myelomonocytic progenitors and the function of macrophages. Blood 2000;96:1723-1732.

$48 \mathrm{Xu} \mathrm{A}$, Wang $\mathrm{Y}$, Keshaw $\mathrm{H}$, et al. The fat-derived hormone adiponectin alleviates alcoholic and nonalcoholic fatty liver diseases in mice. J Clin Invest 2003;112:91-100.

49 Tilg H, Diehl AM. Cytokines in alcoholic and nonalcoholic steatohepatitis. N Engl J Med 2000;343: 1467-1476.

50 Gardner OS, Dewar BJ, Graves LM. Activation of mitogen-activated protein kinases by peroxisome proliferator-activated receptor ligands: an example of nongenomic signaling. Mol Pharmacol 2005;68: 933-941. 